Pacific Journal of Mathematics

ON BOUNDARY BEHAVIOR OF THE BERGMAN KERNEL 


\section{ON BOUNDARY BEHAVIOR OF THE BERGMAN KERNEL FUNCTION AND RELATED DOMAIN FUNCTIONALS}

\section{Bruce L. Chalmers}

Bergman and Hörmander have studied the boundary behavior of the Bergman kernel function for domains in $\underset{\sim}{C^{n}}$, $2 \leqq n<\infty$, with strictly pseudo-convex boundaries. It is the purpose of this paper to extend these results to a larger and more general class of domains with so called strictly $(p, q)$ pseudo-convex boundaries.

Let $B$ be a bounded domain in the space $C^{n}$ of $n$ complex variables, say $z_{k}=x_{k}+i y_{k}, k=1,2, \cdots, n$, where $2 \leqq n<\infty$. (The euclidean norm of a point $z=\left(z_{1}, z_{2}, \cdots, z_{n}\right)$ will be denoted by

$$
|z|=\left(\left|z_{1}\right|^{2}+\cdots+\left|z_{n}\right|^{2}\right)^{1 / 2}
$$

and we let $\bar{z}=\left(\bar{z}_{1}, \bar{z}_{2}, \cdots, \bar{z}_{n}\right)$ and $0=(0,0, \cdots, 0)$.) The space $H(B)$ of all square-integrable holomorphic functions on $B$ forms a Hilbert space with the Bergman (reproducing) kernel function $K_{B}(z, \bar{t})$ with respect to the inner product $(f, g)=\int_{B} f \bar{g} d \omega$, where $f, g \in H(B)$ and $d \omega$ is the euclidean volume element of $B$. If $\left\{\varphi_{n}(z)\right\}_{n=1}^{\infty}$ is any complete orthonormal system for $H(B)$, then

$$
K_{B}(z, \bar{t})=\sum_{n=1}^{\infty} \varphi_{n}(z) \overline{\varphi_{n}(t)},
$$

which converges uniformly in the interior of $B \times B$. Then, if $z^{*}=z^{*}(z)$ is a biholomorphic map from $B$ to $B^{*} \subset \underset{\sim}{C^{n}}$,

$$
K_{B}(z, \bar{t})=K_{B *}\left(z^{*}(z), \overline{t^{*}(t)}\right) \frac{\partial\left(z^{*}\right)}{\partial(z)} \frac{\overline{\partial\left(t^{*}\right)}}{\partial(t)},
$$

and we say $K_{B}(z, \bar{t})$ is a relative (pseudoconformal) invariant. Furthermore

$$
K_{B}(z, \bar{z})=\sup \left\{|f(z)|^{2}: f \in H(B),\|f\| \leqq 1\right\}
$$

is a decreasing domain functional; i.e.,

$$
B \subset A \text { implies } K_{A}(z, \bar{z}) \leqq K_{B}(z, \bar{z}) .
$$

In [1] and [10] respectively Bergman and Hörmander have investigated the behavior of $K(B ; z)=K_{B}(z, \bar{z})$ near a point $Q \in \partial B$, the boundary of $B$, where $B$ is strictly pseudoconvex at $Q$. In each case sufficient conditions were attained for $K(B ; z)$ to become infinite of order $n+1$ at $Q \in \partial B$ (i.e., $|z-Q|^{n+1} K(B ; z)$ remains between two 
positive finite bounds as $z$ nears $Q$ ) in a certain "nontangential" approach $A$. Both procedures use as "basic" domains the complex $n$ dimensional hypersphere $[|z|<1]$ whose kernel function is known and becomes infinite of order $n+1$ at the boundary.

We here introduce an extension of the notion of strictly pseudoconvex as follows:

Definition. Let $B$ be a domain in ${\underset{\sim}{n}}^{n}$. Suppose that there exists an analytic change of coordinates, one-to-one in a neighborhood $\eta$ of $Q \in \partial B$, so that, with respect to the new (normal) coordinates, $Q=0$ and, in a neighborhood of $0, B=\left[2 x_{1}>r_{1(q-1)}^{2}+r_{q n}^{2 / p}+o\left(r_{1(q-1)}^{2}+r_{q n}^{2 / p}\right)\right]$, where $p>0,2 \leqq q \leqq n$, and $r_{u v}^{2}=\sum_{k=u}^{v}\left|z_{k}\right|^{2}$. Then $B$ is said to be strictly $(p, q)$-pseudo-convex at $Q$. If also $B \subset \eta$, then $B$ is said to be globally representable (with respect to normal coordinates) at $Q$.

Note that if $B$ is strictly pseudo-convex at $Q$ then $B$ is strictly $(1, n)$-pseudo-convex at $Q$.

As examples of domains in $C^{2}$ which are strictly $(p, 2)$-pseudo-convex at the points of the circle $\left[z_{1}=0,\left|z_{2}\right|=1\right]$ consider the Reinhardt circular domains given by $\left[\left|z_{2}\right|^{2}<R\left(\left|z_{1}\right|^{2 / p}\right)\right]$, where $\left|z_{1}\right|<R_{0}, R$ is decreasing and $R^{\prime}(0) \neq 0$.

In $\S 2$ and $\S 3$ we prove the following extensions of theorems by Hörmander and Bergman respectively:

Theorem A. In ${\underset{C}{n}}^{n}$ let $B$ be a bounded strictly $(p, q)$-pseudoconvex domain at $Q$. Suppose further that $B$ is a domain of holomorphy. Then $K(B ; z)$ becomes infinite of order $p(n-q+1)+q$ in a "nontangential"' (see §1) approach to $Q$.

Theorem B. In ${\underset{\sim}{C}}^{n}$ let $B$ be a bounded strictly $(p, q)$-pseudoconvex globally representable domain at $Q$. Suppose further that passing through $Q$ is a "normal" (see §3) analytic hypersurface lying entirely outside $B$. Then $K(B ; z)$ becomes infinite of order $p(n-q+1)+q$ in a "nontangential" approach to $Q$.

Since $p$ is an arbitrary positive number, these theorems provide sufficient conditions for the kernel function to become infinite of any order $r>2$. Setting $p=1$ and $q=n$ in Theorem A yields Hörmander's result, while taking $p=1$ and $q=n=2$ in Theorem B gives Bergman's result.

The proofs of Theorems A and B will use as "basic" domains the nonhomogeneous (if $p \neq 1$ ) domains $R_{p}(n, s)=\left[r_{1(s-1)}^{2 / p}+r_{s n}^{2}<1\right]$ which we introduce in $\S 1$. There we will determine the kernel function of these domains and show that it becomes infinite of order $p(n-q+1)+q$, $q=n-s+2$, at the points on the boundary given by $r_{1(s-1)}=0$. 
Under the hypotheses of Theorem B, we will determine in $\S 4$ the boundary behavior of other domain functionals related to the kernel function $K$, such as the Bergman metric given by $d s^{2}=T^{\nu \bar{\mu}} d z_{\nu} d \bar{z}_{\mu}$, where $T^{\bar{\nu}}=\partial^{2} \log K / \partial z_{\nu} \partial \bar{z}_{\mu}$, and the scalar (pseudoconformal) invariant $I=$ $K / \operatorname{det}\left(T^{\nu \bar{\mu}}\right)$.

1. Determination of the Bergman kernel function and its boundary behavior for the domains $R_{p}(n, s)$.

Notation. If $f(x) \in C^{k}$, then denote the $k$-th partial derivative of $f(x)$ with respect to $x$ by $[f(x)]_{(x, k)}$.

THEOREM 1.1. For the kernel function of $R=R_{p}(n, s)$ we have

$$
K(R ; z)=\frac{\left[\left[t^{n \prime}\left(1-t^{p}\right)^{-1}\right]_{\left(t, n-s^{\prime}\right)}\right]_{\left.t^{p}, s^{\prime}\right)}}{\pi^{n}\left(1-r_{s i n}^{2}\right)^{n^{\prime}+1}},
$$

where $t=r_{1}^{2 / p}(s-1)\left(1-r_{s n}^{2}\right)^{-1}, s^{\prime}=s-1, p^{\prime}=p-1$, and $n^{\prime}=n+p^{\prime} s^{\prime}$.

Proof. The monomials $z^{m}=z_{1}{ }^{m_{1}} z_{2}{ }^{m_{2}} \cdots z_{n}{ }^{m_{n}}, m=\left(m_{1}, m_{2}, \cdots, m_{n}\right) \geqq 0$ form a complete set of orthogonal functions in $H(R)$. Thus by $(0.1)$ $K(z)=K(R ; z)=\sum_{m \geqq 0} c_{m}\left|z^{m}\right|^{2}$, where $c_{m}^{-1}=\int_{R}\left|z^{m}\right|^{2} d \omega$. It is clear that $R$ admits the group $G_{1}$ of unitary transformations of the first $s-1$ variables $z_{1}, \cdots, z_{s-1}$ and the group $G_{2}$ of unitary transformations of the last $n-s+1$ variables $z_{s}, \cdots, z_{n}$. The Jacobian of a unitary transformation has modulus one and so $K(z)=K\left(r_{1(s-1)}, 0, \cdots, 0, r_{s n}, 0\right.$,

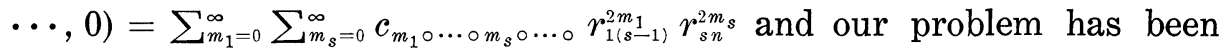
reduced to only a double sum. After a straightforward calculation we find

$$
\begin{aligned}
& c_{m_{1} \circ \cdots \circ m_{s} \circ \cdots \circ}^{-1}{ }^{\prime} \\
& \quad=\frac{\pi^{n} m_{1} ! m_{s} !}{\left(s+m_{1}-1\right) !\left[p\left(s+m_{1}-1\right)+1\right] \cdots\left[p\left(s+m_{1}-1\right)+m_{s}+n-s+1\right]} .
\end{aligned}
$$

Now

$$
\begin{gathered}
\sum_{m_{s}=0}^{\infty} \frac{\alpha(\alpha+1) \cdots\left(\alpha+m_{s}-1\right)}{m_{s} !}\left(r_{s n}^{2}\right)^{m_{s}}=\frac{1}{\left(1-r_{s n}^{2}\right)^{\alpha}}, \text { where } \\
\alpha=p\left(s+m_{1}-1\right)+n-s+2 .
\end{gathered}
$$

Thus,

(2) $K(z)=\frac{1}{\pi^{n}\left(1-r_{s n}^{2}\right)^{n+1}} \sum_{m=0}^{\infty}(m+1) \cdots\left(m+s^{\prime}\right)\left[p\left(m+s^{\prime}\right)+1\right] \cdots$

$$
\cdots\left[p\left(m+s^{\prime}\right)+n-s^{\prime}\right]\left(t^{p}\right)^{m} \text {. }
$$


Now if $Q$ is a boundary point of $R_{p}(n, s)$ with $r_{1(s-1)}=0$, then by a unitary transformation in $G_{2}$ followed by a translation, we can effect an analytic change of coordinates with respect to which $Q=0$ and the domain can be written as

$$
R_{p}^{\prime}(n, s)=\left[2 x_{1}>r_{1(q-1)}^{2}+r_{q n}^{2 / p}\right], \quad q=n-s+2,
$$

with kernel function (obtained from formulas (2) and (0.2)):

$$
K\left(R_{p}^{\prime}(n, s) ; z\right)=\frac{1}{\pi^{n}\left(2 x_{1}-r_{1(q-1)}^{2}\right)^{p(s-1)+q}} \sum_{m=0}^{\infty} a_{m}\left[\frac{r_{q n}^{2}}{\left(2 x_{1}-r_{1(q-1)}^{2}\right)^{p}}\right]^{m},
$$

where the $a_{m}$ are given in (2).

Notation. We shall write $\lim _{z \rightarrow 0}^{A}$, or sometimes simply $\lim ^{A}$, to indicate a limit is being taken as $z \rightarrow 0$ in the set

$$
\left[0<\beta<\frac{x_{1}}{|z|}\right] \text { if } p<2 \text {, and }\left[0<\beta<\frac{x_{1}^{p}}{|z|}\right] \text { if } p \geqq 2,
$$

and say $z \rightarrow 0$ via an $A$-approach. (If for a strictly $(p, q)$-pseudoconvex domain the $z$-coordinates are normal, such an approach to 0 is nontangential in the usual sense.)

Thus from (4) we have

THEOREM 1.2. $K\left(R_{p}^{\prime}(n, s) ; z\right)$ becomes infinite of order $p(n-q+1)+q$ at 0 in a "nontangential" A-approach; in fact

(6) $\lim _{z \rightarrow 0}^{A}\left(2 x_{1}\right)^{p s t+q} K\left(R_{p}^{\prime}(n, s) ; z\right)=\pi^{-n} s^{\prime} !\left[p s^{\prime}+1\right] \cdots\left[p s^{\prime}+q^{\prime}\right]=L$.

2. Poof of Theorem A. The following lemma is due to Hörmander (see [10], p. 146):

Lemma 2.1. (Hörmander) Let $B$ be a bounded domain of holomorphy. Let $Q \in \partial B$ and assume that for some neighborhood $U$ of $Q$ there is an analytic function $u_{0}$ in $B^{\prime}=B \cap U$ such that $\left|u_{0}\right| \leqq 1$ in $B^{\prime},\left|u_{0}(z)\right| \rightarrow 1$ when $z \rightarrow Q$, and $\left|u_{0}(z)\right|$ has an upper bound $<1$ in $B^{\prime} \cap \sim U_{0}$ for some neighborhood $U_{0}$ of $Q$ with compact closure contained in $U$. Then $K(B ; z) / K\left(B^{\prime} ; z\right) \rightarrow 1, z \rightarrow Q$.

Lemma 2.2. $R_{p}(n, s)$ is a domain of holomorphy.

Proof. It follows easily from (1.1) that $K\left(R_{p}(n, s) ; z\right)$ becomes infinite at every boundary point of $R_{p}(n, s)$. But it is well known that a domain whose kernel function becomes infinite at every boundary point is a domain of holomorphy (see e.g., [7], p. 357). 
Lemma 2.3. If $B$ is strictly $(p, q)$-pseudo-convex at $Q$, then, with respect to local normal coordinates

$$
\varlimsup^{A}\left(2 x_{1}\right)^{p s^{\prime}+q} K(B ; z) \leqq L .
$$

Proof. $B$ can be written locally in a neighborhood of $Q=0$ as $B=\left[2 x_{1}>r_{1(q-1)}^{2}+r_{q n}^{2 / p}+o\left(r_{1(q-1)}^{2}+r_{q n}^{2 / p}\right)\right]$. For $\varepsilon>0$ let

$$
R_{\varepsilon}=\left[2 x_{1}>r_{1(q-1)}^{2}+r_{q n}^{2 / p}+\varepsilon\left(r_{1(q-1)}^{2(}+r_{q n}^{2 / p}\right)\right] \text {. }
$$

Then $R_{\varepsilon}^{\delta}=R_{\varepsilon} \cap[|z|<\delta]$ is included in $B$ if $\delta$ is sufficiently small. Since $R_{s}$ is obtained from $R_{p}^{\prime}(n, s)$ by the change of coordinates $z_{k} \leftarrow(1+\varepsilon)^{-1} z_{k}, k=1, \cdots, q-1, z_{l} \leftarrow(1+\varepsilon)^{-p} z_{l}, \quad l=q, \cdots, n, R_{s} \quad$ is also a domain of holomorphy. Thus

$$
\begin{array}{r}
\varlimsup_{\lim ^{A}}\left(2 x_{1}\right)^{p s f+q} K(B ; z) \leqq \varlimsup_{\lim ^{A}}\left(2 x_{1}\right)^{p_{s} \prime+q} K\left(R_{\varepsilon}^{\delta} ; z\right) \\
=\varlimsup_{\lim ^{A}}\left(2 x_{1}\right)^{p_{s}+q} K\left(R_{\varepsilon} ; z\right)=(1+\varepsilon)^{p_{s}+q-2} L
\end{array}
$$

by (0.4), Lemma 2.1 (taking $u_{0}(z)=e^{-z_{1}}$ and $U=[|z|<\delta]$ ), and (0.2) together with Theorem 1.2, respectively.

Conclusion of proof of Theorem $A$. Let $0<\varepsilon<1$. Then, analogously as in the proof of Lemma 2.3., $B^{\delta}=B \cap[|z|<\delta] \subset R_{-s}$ if $\delta$ is sufficiently small. Now since $B$ is a domain of holomorphy we can apply Lemma 2.1 with $u_{0}(z)=e^{-z_{1}}$ and $U=[|z|<\delta]$ to obtain

$$
\begin{aligned}
& \underline{\lim }\left(2 x_{1}\right)^{p s^{\prime \prime q}} K(B ; z)=\underline{\lim }\left(2 x_{1}\right)^{p s^{\prime+q}} K\left(B^{\hat{o}} ; z\right) \geqq \underline{\lim }\left(2 x_{1}\right)^{p s^{\prime}+q} K\left(R_{-s} ; z\right) \\
& \quad=(1-\varepsilon)^{p s^{\prime \prime+q-2}} L,
\end{aligned}
$$

using also (0.4) and (0.2) together with Theorem 1.2. Combining this result with that of Lemma 2.3 , we conclude that under the hypotheses of Theorem A,

$$
\lim ^{A}\left(2 x_{1}\right)^{p(n-q+1)+q} K(B ; z)=L .
$$

\section{Proof of Theorem B.}

Definition 3.1. Suppose that $B$ is a strictly $(p, q)$-pseudo-convex globally representable domain at $Q$. Suppose $h$ is an analytic hypersurface through $Q$ which can be represented as $h=\left[z_{1}=0\right]$ with respect to normal coordinates for $B$ and $h \cap \bar{B}=Q$. Then we say that through $Q$ passes the "normal" analytic hypersurface $h$ lying entirely outside $B$.

LEMma 3.2. Let $B$ be a bounded domain whose closure intersects the hyperplane $\left[z_{1}=0\right]$ only at the origin. Then an arbitrary neigh- 
borhood of the origin includes the section $B \cap\left[z_{1}=\gamma\right]$ if $\gamma$ is sufficiently small.

Proof. Let $P=\sup _{z \in B} r_{2 n}$. Since the set of points $\left[z_{1}=0, b \leqq r_{2 n} \leqq P\right]$ is closed, for each $b>0$ there exists a number $\tau(b), 0<\tau(b) \leqq b$, such that $\left[\left|z_{1}\right| \leqq \tau(b), b \leqq r_{2 n} \leqq P\right]$ contains no point of $B$. Now if $U$ is any neighborhood of the origin, there is a $\nu>0$ such that $\left[\left|z_{1}\right|<\nu\right.$, $\left.r_{2 n}<\nu\right] \subset U$. Then if $z \in B$ and $b \leqq \nu$, we have that $\left|z_{1}\right|<\tau(b)$ implies $r_{2 n}<b$, and so the section $B \cap\left[z_{1}=\gamma\right] \subset U$ for $|\gamma|<\tau(b)$.

Lemma 3.3. Let $B$ be a domain satisfying the hypotheses of Theorem $B$. Then there exists a transformation

$$
W: z_{1} \leftarrow \frac{z_{1}}{1-\alpha z_{1}}, z_{k} \leftarrow z_{k} \frac{1+(\beta-\alpha) z_{1}}{1-\alpha z_{1}}, k=2, \cdots, n
$$

such that for $\alpha$ and $\beta$ positive and sufficiently large, the domain $R_{-\varepsilon}^{\alpha \beta}=W\left(R_{-\varepsilon}\right)$ includes $B$ in its interior, where $0<\varepsilon<1$.

Proof.

$$
B=\left[2 x_{1}>r_{1(q-1)}^{2}+r_{q n}^{2 / p}+o\left(r_{1(q-1)}^{2}+r_{q n}^{2 / p}\right)\right] \text { and }
$$

$R_{-\varepsilon}=\left[2 x_{1}>(1-\varepsilon)\left(r_{1(q-1)}^{2}+r_{q n}^{2 / p}\right)\right]$. Thus $\exists$ a neighborhood $\eta$ of 0 such that $B \cap \eta \subset R_{-\varepsilon} \cap \eta$. Hence for $|\gamma|<\delta, B \cap\left[z_{1}=\gamma\right] \subset R_{-\varepsilon} \cap\left[z_{1}=\gamma\right]$ if $\delta$ is sufficiently small, by Lemma 3.2.

Now let $R_{-\varepsilon}^{\alpha}=W^{\alpha}\left(R_{-\varepsilon}\right)$, where $W^{\alpha}: z_{1} \leftarrow z_{1} /\left(1-\alpha z_{1}\right), z_{k} \leftarrow z_{k}, k=$ $2, \cdots, n$. Then consider $R_{-\varepsilon}^{\alpha} \cap\left[r_{2 n}=0\right]=\sim c_{\alpha}$, where $c_{\alpha}$ is the circle

$$
\left[\left|z_{1}+\frac{1}{2 \alpha-(1-\varepsilon)}\right| \leqq \frac{1}{2 \alpha-(1-\varepsilon)}\right] \text {. }
$$

We can assume $\alpha$ is so large that $c_{\alpha} \cap \bar{B} \cap\left[r_{2 n}=0\right]=0$. Then the closed set $M=\bar{B} \cap\left[r_{2 n}=0\right] \cap\left[\left|z_{1}\right| \geqq \delta\right]$ lies entirely in the interior of the section $R_{-s}^{\alpha} \cap\left[r_{2 n}=0\right]$. Now the sections $R_{-\varepsilon}^{\alpha} \cap\left[z_{1}=\gamma\right], \gamma \in M$, include hyperspheres with center $(\gamma, 0, \cdots, 0)$ and the radii of these hyperspheres have a positive lower bound $r$. If one applies to $R_{-\varepsilon}^{\alpha}$ the transformation $W_{\beta}: z_{1} \leftarrow z_{1}, z_{k} \leftarrow z_{k}\left(1+\beta z_{1}\right), k=2, \cdots, n$, to obtain the domain $R_{-\varepsilon}^{\alpha \beta}=W_{\beta}\left(R_{-\varepsilon}^{\alpha}\right)$, then the section $R_{-\varepsilon}^{\alpha} \cap\left[z_{1}=\gamma\right]$ goes into a domain including a hypersphere with center $(\gamma, 0, \cdots, 0)$ and radius $\geqq r|1+\beta \gamma|$. $\beta$ can be chosen so large that $r|1+\beta \gamma|>P$ for $|\gamma| \geqq \delta$, where $P=\sup _{z \in B} r_{2 n}$. Thus $B \cap\left[z_{1}=\gamma\right] \subset R_{-\varepsilon}^{\alpha \beta} \cap\left[z_{1}=\gamma\right]$ if $|\gamma| \geqq \delta$.

On the other hand consider $\gamma \in R_{-\varepsilon}^{\alpha} \cap\left[r_{2 n}=0\right]=\sim c_{\alpha}$. Thus if $\beta>2 \alpha-(1-\varepsilon)$, then $|\gamma+1 / \beta|>1 / \beta$ and so $|1+\beta \gamma|>1$. Hence $R_{-\varepsilon}^{\alpha \beta} \cap\left[z_{1}=\gamma\right] \supset R_{-\varepsilon}^{\alpha} \cap\left[z_{1}=\gamma\right]$. But for $|\gamma|<\delta$ we know $R_{-\varepsilon}^{\alpha} \cap\left[z_{1}=\right.$ $\gamma] \supset B \cap\left[z_{1}=\gamma\right]$. Thus $R_{-\varepsilon}^{\alpha \beta} \cap\left[z_{1}=\gamma\right] \supset B \cap\left[z_{1}=\gamma\right]$ for all $\gamma$. 
Conclusion of proof of Theorem $B$. Since $R_{-\varepsilon}^{\alpha \beta} \supset B$, we have $\underline{\lim }^{A}\left(2 x_{1}\right)^{p s t+q} K(B ; z) \geqq \underline{\lim }^{A}\left(2 x_{1}\right)^{p s \prime+q} K\left(R_{-\varepsilon}^{\alpha \beta} ; z\right)=(1-\varepsilon)^{p s \prime+q-2} L$. This result together with Lemma 2.3 shows that (2.2) also holds for $B$ under the hypotheses of Theorem $B$.

4. Boundary behavior of other functionals on the domains of Theorem $B$.

THEOREM 4.1. Under the hypotheses of Theorem $B$ and using normal coordinates, we have further, denoting $\lim ^{A}\left(2 x_{1}\right)^{2} F(B ; z)$ by $(\lambda, F)$ and letting $m=p(q-1)$ and $\alpha=m+s$,

$$
\begin{aligned}
& \left(\alpha+1, K_{z_{1}}\right)=-L \alpha ;\left(\alpha+1 / 2, K_{z_{k}}\right)=0,2 \leqq k \leqq q-1 ; \\
& \left(\alpha+p / 2, K_{z_{k}}\right)=0, q \leqq k \leqq n \\
& \left(2, d s^{2}\right)=\alpha\left|d z_{1}\right|^{2} \text { if } 0<p<2 ; \\
& \left(2, d s^{2}\right)=\alpha\left|d z_{1}\right|^{2}+c \sum_{k=s}^{n}\left|d z_{k}\right|^{2} \text { if } p=2, \\
& \left(p, d s^{2}\right)=c \sum_{k=s}^{n}\left|d z_{k}\right|^{2} \text { if } p>2, \text { where } \\
& \quad c=q(p+m+1) \cdots(p+\alpha-1) /[(m+1) \cdots(\alpha-1)],
\end{aligned}
$$

( 3 ) $(o, I)$

$$
=\pi^{-n}(q-1) ! \alpha^{-n-1}[(m+1) \cdots \alpha]^{q}\left[q(p+m+1) \cdots(p+\alpha-1]^{1-q} .\right.
$$

Proof (Sketch). One can show analogously as in Lemma 3.3 that $B$ includes in its interior the domain $R_{\varepsilon}^{\alpha \beta^{\prime}}=W\left(R_{\varepsilon}\right)$ where

$$
W: z_{1} \leftarrow \frac{z_{1}}{1+\alpha^{\prime} z_{1}}, z_{k} \leftarrow \frac{z_{k}\left(1+\alpha^{\prime} z_{1}\right)}{1+\left(\alpha^{\prime}+\beta^{\prime}\right) z_{1}}, k=2, \cdots, n,
$$

for sufficiently large $\alpha^{\prime}$ and $\beta^{\prime}$. Then if $F(B ; z)$ is any decreasing functional of the domain $B$, e.g., $K(B ; z)$, we have

$$
F\left(R_{\varepsilon}^{\alpha / \beta \prime} ; z\right) \geqq F(B ; z) \geqq F\left(R_{-\varepsilon}^{\alpha \beta} ; z\right) .
$$

It is well known that the quantities $d s^{2}$ and $I$ are rational functions of decreasing domain functionals $F_{i}$ which can be determined from the kernel function and its derivatives. But from our results in $\S 1$, and using (0.2), we can calculate the kernel function and its derivatives for the domains $R_{\varepsilon}^{\alpha \beta \beta \prime}$ and $R_{-s}^{\alpha \beta}$. Thus we can calculate the decreasing domain functionals $F_{i}$ and show that there exist $\lambda_{i}$ such that $\lim _{\varepsilon \rightarrow 0}\left(\lambda_{i}, F_{i}\left(R_{\varepsilon}^{\alpha \beta \beta \prime} ; z\right)\right)=\lim _{\varepsilon \rightarrow 0}\left(\lambda_{i}, F_{i}\left(R_{-\varepsilon}^{\alpha \beta} ; z\right)\right)=l_{i}$, whence $\left(\lambda_{i}, F_{i}(B ; z)\right)=l_{i}$. From these results (2) and (3) follow immediately. Although the derivatives $K_{z_{k}}$ are not themselves rational functions of monotone domain 
functionals, they can be approximated above and below by such functions and the same method yields (1).

The author wishes to express his thanks to Professor S. Bergman for his guidance and to Professor C. Loewner and M. Skwarczynski for their pertinent criticism in the preparation of this paper.

\section{BIBLIOGRAPHY}

1. S. Bergman, Uber die Kernfunktion eines Bereiches und ihr Verhalten am Rande, J. Reine Angew. Math. 169 (1933), 1-42; and 172 (1934), 89-128.

2. The behaviour of the kernel function at boundary points of the second order, Amer. J. Math. 65 (1943), 679-700.

3. - Sur les functions orthogonales de plusieurs variables complexes, Mém. des Sciences Math. 106 (1947).

4. - Sur la fonction-noyau d'un domaine et ses applications dans la théorie des transformations psuedo-conformes, Mém. des Sciences Math. 108 (1948).

5 . - The kernel function and conformal mapping, Math. Surveys 5, Amer. Math. Soc., New York, 1950.

6. — Z Zur Theorie von Pseudokonformen Abbildungen, Recueil Math. 1 (1936), 79-96.

7. H.J. Bremermann, "Holomorphic continuation of the kernel function and the Bergman metric in several complex variables,"in Lectures on functions of a complex variable, ed. by W. Kaplan, Univ. of Michigan press, 1955.

8. K. Diederich, Das Rand verhalten der Bergmanschen Kernfunktion und Metrik in streng pseudokonvexen Gebieten (to appear)

9. B. A. Fuks, Special chapters in the theory of analytic functions of several complex variables, (Russian) Moscow, 1963 (Engl. Transl., A.M.S. 1965).

10. L. Hörmander, Existence theorem for the $\delta$-operator by $L^{2}$ methods, Acta Math. 113 (1965), 89-152.

11. H. Meschkowski, Hilbertsche Räume mit Kernfunktion, Berlin, 1962.

Received June 24, 1968, and in revised form August 19, 1968. This paper includes the essential contents of the author's doctoral thesis at Stanford University.

UNiversity of California, Riverside 


\section{PACIFIC JOURNAL OF MATHEMATICS}

\section{EDITORS}

H. ROYDEN
Stanford University
Stanford, California

\section{R. R. PHELPS}

University of Washington

Seattle, Washington 98105
J. DUGUNDJI

Department of Mathematics

University of Southern California

Los Angeles, California 90007

\section{RICHARD ARENS}

University of California

Los Angeles, California 90024

\section{ASSOCIATE EDITORS}

E. F. BeCKenbaCh
B. H. NEUMANN

F. WOLF
K. YoshidA

\section{SUPPORTING INSTITUTIONS}

\author{
UNIVERSITY OF BRITISH COLUMBIA \\ CALIFORNIA INSTITUTE OF TECHNOLOGY \\ UNIVERSITY OF CALIFORNIA \\ MONTANA STATE UNIVERSITY \\ UNIVERSITY OF NEVADA \\ NEW MEXICO STATE UNIVERSITY \\ OREGON STATE UNIVERSITY \\ UNIVERSITY OF OREGON \\ OSAKA UNIVERSITY \\ UNIVERSITY OF SOUTHERN CALIFORNIA
}

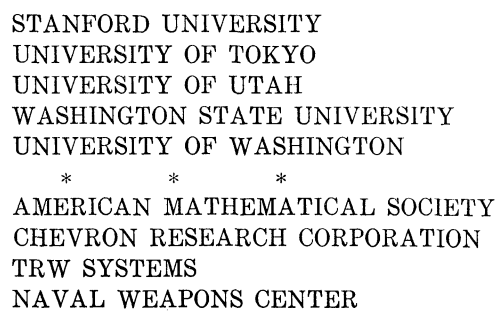

The Supporting Institutions listed above contribute to the cost of publication of this Journal, but they are not owners or publishers and have no responsibility for its content or policies.

Mathematical papers intended for publication in the Pacific Journal of Mathematics should be in typed form or offset-reproduced, double spaced with large margins. Underline Greek letters in red, German in green, and script in blue. The first paragraph or two must be capable of being used separately as a synopsis of the entire paper. It should not contain references to the bibliography. Manuscripts, in duplicate if possible, may be sent to any one of the four editors. Please classify according to the scheme of Math. Rev. 36, 1539-1546. All other communications to the editors should be addressed to the managing editor, Richard Arens, University of California, Los Angeles, California, 90024.

50 reprints are provided free for each article; additional copies may be obtained at cost in multiples of 50 .

The Pacific Journal of Mathematics is published monthly. Effective with Volume 16 the price per volume (3 numbers) is $\$ 8.00$; single issues, $\$ 3.00$. Special price for current issues to individual faculty members of supporting institutions and to individual members of the American Mathematical Society: $\$ 4.00$ per volume; single issues $\$ 1.50$. Back numbers are available.

Subscriptions, orders for back numbers, and changes of address should be sent to Pacific Journal of Mathematics, 103 Highland Boulevard, Berkeley, California, 94708.

PUBLISHED BY PACIFIC JOURNAL OF MATHEMATICS, A NON-PROFIT CORPORATION

Printed at Kokusai Bunken Insatsusha (International Academic Printing Co., Ltd.), 7-17, Fujimi 2-chome, Chiyoda-ku, Tokyo, Japan. 


\section{Pacific Journal of Mathematics \\ Vol. 29, No. $2 \quad$ June, 1969}

Bruce Langworthy Chalmers, On boundary behavior of the Bergman kernel function and related domain functionals ................... 243

William Eugene Coppage, Peirce decomposition in simple Lie-admissible power-associative rings .............................. 251

Edwin Duda, Compactness of mappings...................... 259

Earl F. Ecklund Jr., On prime divisors of the binomial coefficient......... 267

Don E. Edmondson, A modular topological lattice ............... 271

Phillip Alan Griffith, A note on a theorem of Hill ................... 279

Marcel Herzog, On finite groups with independent cyclic Sylow

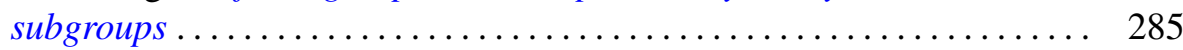

James A. Huckaba, Extensions of pseudo-valuations................. 295

S. A. Huq, Semivarieties and subfunctors of the identity functor ........ 303

I. Martin (Irving) Isaacs and Donald Steven Passman, Finite groups with small character degrees and large prime divisors. II ............ 311

Carl Kallina, A Green's function approach to perturbations of periodic

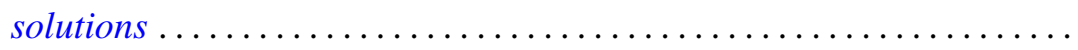

$\mathrm{Al}$ (Allen Frederick) Kelley, Jr., Analytic two-dimensional subcenter manifolds for systems with an integral ....................

Alistair H. Lachlan, Initial segments of one-one degrees ............ 351

Marion-Josephine Lim, Rank k Grassmann products ............. 367

Raymond J. McGivney and William Henry Ruckle, Multiplier algebras of

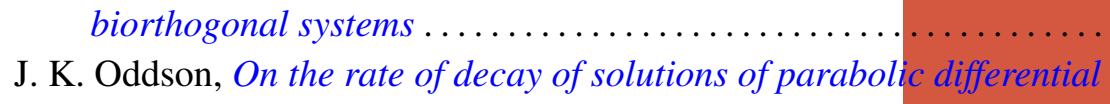

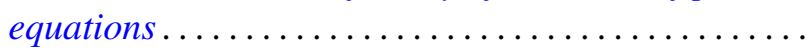

Helmut R. Salzmann, Geometries on surfaces ........... .

Annemarie Schlette, Artinian, almost abelian groups and their groups of automorphisms ............................

Edgar Lee Stout, Additional results on modules over polydisc algebras ...

Lajos Tamássy, A characteristic property of the sphere . .

Mark Lawrence Teply, Some aspects of Goldie's torsion theory. ...

Freddie Eugene Tidmore, Extremal structure of star-shaped sets ...

461

Leon Jarome Weill, Unconditional and shrinking bases in locally convex spaces... 